\title{
Seroprevalence of Hepatitis B Virus among Human Immunodeficiency Virus patients attending tertiary and secondary health facilities in Benin City Nigeria.
}

\author{
E.U. Eze, A.N. Onunu, E.P. Kubeyinje
}

\begin{abstract}
The HIV/AIDS pandemic has changed the prevalence of some infectious diseases. Hepatitis B is a very important potentially lethal and presently treatable infection which affects the course of HIV disease. This study was carried out to determine the prevalence of hepatitis B virus [HBV] infection in human immunodeficiency virus (HIV) infected patents undergoing treatment in two major hospitals in Edo state over a 12 month period. All HIV infected persons aged 15 years and above of either gender seen between $1^{\text {st }}$ January and $31^{\text {st }}$ December 2002 in the University of Benin Teaching hospital and central Hospital Benin City were included in this study. A total of 204 HIV infected patients [81males, 123 females] seen over this period were screened for HbsAg. HIV infection was confirmed using the double ELISA technique. One hundred and sixty six [66 males and 100 females] age and sex matched HIV negative patients recruited from the general outpatients clinic of those hospitals formed the control group. The mean age of HIV infected patients was 37.14 \pm 10.94 with a range of 15-70. The two groups compared well as regards age, sex, occupation and social class. However, widows has significantly higher prevalence amongst the HIV patients. Seventy one percent [71\%] of the HIV patients were symptomatic. The prevalence of HBV amongst the HIV infected patients was $10.75 \%$ as compared to $7.2 \%$ in the control [P>0.05]. This was however not statistically significant. This study showed that though the prevalence of Hepatitis B infection is higher in HIV infected patients, this difference is however, not statistically significant from that in HIV negative controls.
\end{abstract}

Running title: prevalence of hepatitis B infection in HIV infected persons

\section{INTRODUCTION}

The prevalence of several diseases has changed with the advent of human

KEYwORDS: Hepatitis B infection, HIV infection, Benin City.

Department of Medicine University of Benin teaching Hospital P.M.B. 1111 Benin City, Nigeria.

Correspondence: Dr. E.U. Eze Department of medicine University of Benin teaching hospital P.M.B 1111 Benin City, Nigeria. E-mail; Phone; 08034280193 immunodeficiency virus (HIV) pandemic. The incidence and prevalence of some infectious diseases that were hitherto on the decline are now experiencing an upward trend1-5. Hepatitis B (HBV) is a very important potentially lethal and presently treatable disease. This on its own may be a significant cause of morbidity and mortality. ${ }^{5}$ The prevalence rate of $\mathrm{HBV}$ infection has been shown to be higher in HIV infected persons than the general population ${ }^{6,7}$. The 
presence of hepatitis $B$ in HIV infected patients may pose serious problems for them.

The prevalence of HBV varies significantly from one geographical region to another. It is highly endemic in south East Asia, China and Sub-Saharan Africa with carrier rates of 8-20 percent. ${ }^{7}$ In these regions $70-90 \%$ of the population show serologic evidence of previous and current HBV infection. In the general population, the prevalence is between $0.5-2$ percent. In Nigerian children, a prevalence of $10 \%$ has been reported ${ }^{7}$. Amongst Intravenous (IV) drugs users and HIV infected persons the prevalence is higher ${ }^{5}$.

Transmission of HBV and HIV infection is quite similar. Both infections are transmitted horizontally and vertically. Horizontally, transmission occurs via coitus, percutaneous and parenteral routes such as transfusion of contaminated blood or blood products, needlestick injuries, intravenous (I.V) drug abuse, dental and surgical procedures, hemodialysis, tattooing, acupuncture, circumcision and ear piercing. Vertical transmission occurs from infected mother to infants in the uterus or during the perinatal or postnatal period. ${ }^{7,8}$

The incubation period of this infection averages about 3 months, with a transient viraemia lasting 4-8 weeks. In about $90 \%$ of patients, the acute infection is silent. The three antigens hepatitis B surface antigen (HbsAg), hepatitis B core antigen ( $\mathrm{HbcAg}$ ) and Hepatitis B infectivity antigen (HbeAg) elicit an antibody response ${ }^{8}$. Only Anti-HBS accounts for recovery and immunity ${ }^{7,8}$. Ongoing infectivity and viral replication is suggested by high titer of IgM anti-HbsAg and persistent HbeAg and HBV DNA polymerase. Presence of Anti-HbcAg suggests low infectivity $^{8}$. Rates of progression to cirrhosis and hepatocellular carcinoma vary according to the patients' immune status, age and serologic stage of the infection, as well as geographic and genetic factors. The relative risk of death due to cirrhosis for HbeAg carriers as compared with normal individuals ranges from 12 to 79 and relative risk of hepatocellular carcinoma ranges from 148 in Alaska to 30-98 in the Far East. ${ }^{7,8}$

HIV infection has reached pandemic proportions worldwide with sub Saharan Africa being the worst hit. In Eastern and southern Africa the prevalence ranges from $9 \%$ in males to $34 \%$ in females ${ }^{9}$. Whilst in Nigeria, the prevalence is $4.4 \%{ }^{10}$. It is caused by a retrovirus that attacks the central point in the human immune system, the CD4 + cell, and therefore lowers the patient's, immunity and predisposes the patient to opportunistic infections and secondary malignancies ${ }^{11,12}$.

There is an acute phase of infection which in most cases is silent but about 20\% present with flu-like symptoms and a glandular fever like illness, sometimes aseptic meningitis (stage 1). The infection becomes latent and asymptomatic for 5-8 years (stage II). Then it gets to the stage where in about $10 \%$ of patients there is persistent generalized lymphadenopathy (stage III) and finally AIDS develops(stage IV) with full blown symptoms such as wasting (slim disease) recurrent fever and diarrhoea, numerous skin manifestations, opportunistic diseases and secondary malignancies ${ }^{12}$.

Highly active antiretroviral therapy (HAART) is giving HIV infected patients a new lease of life, therefore, there is need to tackle the health problems associated with HBV in order to reduce the morbidity and mortality in these patients. Also with the use of lamivudine as treatment in patients infected with HBV, the outlook is improving for HIV and HBV co-infected patients.

Studies have been done in Nigeria to determine the prevalence of $\mathrm{HBV}$ in the general population especially amongst certain risk groups such as Health workers, Blood donors, dialysis patients and prisoners, 
but no study has been done amongst HIV infected persons, comparing the findings with controls in this part of the country. The paucity of reports from this region warrants a closer study of these diseases in this environment. Therefore the objective of this study is to determine the seroprevalence of Hepatitis B infection amongst HIV infected persons in Edo state, Nigeria.

\section{PATIENTS AND METHODS}

\section{Patients}

The study was carried out at the University of Benin Teaching Hospital (UBTH) Benin City and the Central Hospital $(\mathrm{CH})$, Benin City, Edo State, Nigeria, between $1^{\text {st }}$ January and $31^{\text {st }}$ December 2002. The University of Benin Teaching Hospital caters for the tertiary Health care needs of patients referred from Edo, Delta, Ekiti, Bayelsa, Kogi and Ondo states. Its coverage serves a population of over 5 million people. The central Hospital Benin city is a secondary health facility in Benin that receives a large bulk of patients due to its centrality of location and low cost of services.Both Hospitals have over 400 beds and are diagnostic and treatment centers for HIV/AIDS and Infectious diseases.

All HIV infected persons aged 15 years and above of either gender seen between $1^{\text {st }}$ January and $31^{\text {st }}$ December 2002 in the University of Benin Teaching Hospital and Central Hospital Benin City were included in this study. Age and sex matched controls were recruited from patients attending the out patients clinics of the above Hospitals who were HIV negative. Patients with sickle cell anemia and all patients with other immunocompromising diseases such as diabetes mellitus and lymphoma were excluded. Clinical assessment included a detailed history and physical examination. Pre-test and Post-test HIV counseling were done for all patients, blood samples were taken and tests were carried out with the informed consent of all patients.

Consecutive sampling was used. Using a prevalence of $6.05 \%{ }^{6}$, sample size for comparative study $^{13}$ per group was 93. However a sample size of 204 patients HIV positive and 166 controls was used.

\section{Laboratory Methods}

Two assays were done for each of the patients: HIV screening by Elisa technique and confirmed by double Elisa and Hepatitis B virus (HbsAg) screening.

\section{Hiv Test}

The tests for HIV were done using ELISA HIV test kits, Determine by Abbott and Stat Pak by CHEMBIO. Confirmation of indeterminate cases was done with GENIE by BIO-RAD.

\section{HbsAg test}

The screening tests for HbsAg were done using the ELISA test kits by Human Gesellschaft for biomedical and diagnostica mbti-mbh max-panch-ing 21-65205 Wiesbauden Germany.

Fasting blood sugar and Hemoglobin electrophoresis were done to rule out diabetes mellitus and sickle cell anemia respectively. Routine full blood counts, electrolytes and urea and liver function tests were also done.

\section{Statistics}

The data were analyzed using the EPI- INFO 6 statistical program. Continuous variables were described with means while discontinuous variables were described with proportions. Group means were compared with two-tailed t-test. When comparing groups of subjects, the $\mathrm{X}^{2}$ (Chi square) test was applied to determine the significance of differences observed. Where the number in any cell was less than five, Fisher exact tests were used. 


\section{Results}

A total of 370 subjects were involved in the study. There were 204 HIV positive patients while another 166 were HIV negative controls .Amongst the HIV positive patients, 81 were males and 123 were females with a mean age of $37.14 \pm 10.94$ years (Age range 15-70years) In the control group 66 were males and 100 were females with a mean age of $37.21 \pm 11.30$ years (Age range 1570 years). There was no significant age and sex difference between HIV positive patients and controls. $(\mathrm{P}>0.05)$. Table 1 shows that the two groups of patients in the study were comparable as regards age and sex.

Table 1: Demographic data of patients and controls

\begin{tabular}{|c|c|c|c|}
\hline Gender & Subjects & Control & $\mathrm{p}$-value \\
\hline Male & 18 & 66 & \\
\hline Female & 123 & 100 & \\
\hline Total & 204 & 166 & \\
\hline \multicolumn{4}{|c|}{ Age (Years) } \\
\hline Mean age & $37.14+10.94$ & $37.21+11.30$ & \\
\hline
\end{tabular}

Table 2: shows the clinical stage I - II of HIV infected patients seen in this study. A total of 145 (71\%) were asymptomatic. Fifty-six (56) were male while 89 were female. Two subjects, both male (1.0\%) had only persistent generalized lymphadenopathy. Fifty-seven (57) patients (27\%) had full blown AIDS; twenty-three (23) who were males while 34 were female.

Table 2: Clinical Staging of HIV infected patients

\begin{tabular}{llll}
\hline Clinical stage & Male & Female & Total(\%) \\
\hline Stage I & 0 & 0 & 0 \\
Stage II & 56 & 89 & $145(71.1)$ \\
Stage III & 2 & 0 & $2(1.0)$ \\
Stage IV & 23 & 34 & $57(27.9)$ \\
\hline Total & 81 & 123 & $204(100)$ \\
\hline
\end{tabular}


Table 3: shows the prevalence of hepatitis B and HIV co-infection compared to the HIV negative controls. Of the 204 HIV infected patients tested, 22(10.8\%) were also hepatitis B infected compared to $12(7.2 \%)$ out of the 166 HIV negative controls. Even through the prevalence of hepatitis B infection is higher in HIV infected patients, this difference is, however, not statistically significant from that in HIV negative controls $(\mathrm{P}>0.05)$. The overall prevalence of Hepatitis B infection in both groups was $9.2 \%$.

Table 3: prevalence of Hepatitis B and HIV Co-infection

\begin{tabular}{|c|c|c|c|}
\hline Hepatitis B status & $\frac{\text { HIV status }}{\text { HIV + (\%) }}$ & HIV-(\%) & Total (\%) \\
\hline Positive & $22(10.8)$ & $12(7.2)$ & $34(9.2)$ \\
\hline Negative & 182(89.2) & $154(92.8)$ & $336(90.8)$ \\
\hline Total & $204(55)$ & $166(45)$ & $370(100)$ \\
\hline
\end{tabular}

\section{Discussion}

HIV disease affects the prevalence and prognosis of HIV infection. In the developed world, the prevalence of HBV infection in the general population is $0.5-2 \%$ but is up to 80 $90 \%$ amongst HIV infected individuals ${ }^{5}$. Ahmed et al in Malawi showed that in pregnant women there was no statistical evidence to suggest that HIV infection was associated with an increased prevalence of HBV markers ${ }^{14}$. The presence of HBV in HIV patients increases the morbidity of HIV disease ${ }^{15}$ and prognosis for HBV is worse in the presence of HIV infection ${ }^{1}$.The hepatitis $B$ virus progresses to chronic carrier state but does not progress to fibrosis and cirrhosis as fast as in the non-HIV infected chronic hepatitis patients. The incidence of chronic carrier state was shown to be forty times higher than in the general population in one study ${ }^{1}$.

In Nigeria, a study done in Jos showed HBV prevalence to be as high as $28.7 \%$ in the HIV infected population ${ }^{16}$ while it was $48 \%$ amongst prisoners in Nigeria ${ }^{17}$.

This study showed that HIV prevalence did not significantly affect the prevalence of hepatitis B in Benin City even though the prevalence was higher. $(10.8 \%$ in HIV- positive versus $7.2 \%$ in HIV negatives.) The figures are in agreement with the literature that showed a prevalence of $8.9 \%$ in $\mathrm{Jos}^{16}$ and $8.6 \%$ amongst hemodialysis patients in a teaching hospital in $\mathrm{Jos}^{18}$. In sub Saharan Africa carrier rates are $8.2 \%$ in published data ${ }^{19,20}$.The prevalence in this study is therefore comparable with other reports, despite the difference between the prevalence in HIV positive and HIV negative patients that is not statistically significant $\left(\mathrm{X}^{2}=0.99\right)$. Report in Nigeria by Idoko and Sirisena ${ }^{16}$ showed a significant difference between the HIV positive and HIV negative groups in Nigeria. This difference is more pronounced in data from developed countries ${ }^{5}$.

The observed lower prevalence of hepatitis B in this study as compared with some other rates in the literature ${ }^{5,16}$ may be 
due to the sample size used compared to other studies. It may be due to lower prevalence of risk factors for $\mathrm{HBV}$ transmission in this study group such as Intravenous drug users (IDUS), ${ }^{5}$ blood products recipients, and hemodialysis patients ${ }^{18}$. The prevalence of HBV in HIV patients in this study though not significantly higher in HIV infected individuals than controls was lower compared to figures reported in Europe and America ${ }^{5}$ In these counties, HIV and HBV coinfection was usually associated with I.V. drugs abuse, which in itself is a major risk factor for $\mathrm{HBV}$ transmission. Intravenous drug abuse is not a common problem in this part of the world and is still not a major route for HIV transmission in Africa. A more prevalent risk factor such as homosexuality in the western world is also a risk factor, which is not as frequently reported in this part of the world ${ }^{5}$. This may explain the comparatively low figures seen in this study.

Other studies focus on the prevalence of $\mathrm{HBV}$ in high risk groups such as commercial sex workers, intravenous drug users, blood products recipients and blood donors. This study was conducted amongst patients who largely did not have these risk factors ${ }^{20,21}$.

The prevalence reported in this study of HBV in HIV patients actually compares with that in the general population as seen in this study. This is in agreement with other studies done both in Nigeria and elsewhere showing that HIV prevalence did not on its own affect the HBV prevalence in this part of the world. The study done in Jos, Nigeria, which showed significant differences were done among prisoners who are a high risk group for homosexual behavior and I.V drug abuse, and among hemodialysis patients who on their own are a high risk group for HBV infection. ${ }^{16-18}$.

The prevalence of Hepatitis B is not significantly higher in HIV positive individuals than in the general population in this study. It is important to note however that the prevalence of hepatitis B in the general population is also quite high. This highlights the need for prevention of this disease. Thus all blood for transfusion should be screened for these infections and standard infection control measures (universal and standard precautions) should be emphasized in all levels of population and especially among healthcare workers in our hospitals.

\section{References}

1. Sullivan P.S. Dworkin M.S. Harrision: D.L. Flerming P.C. Trends in AIDS defining opportunistic illness diagnoses through 1998. Programs and Abstracts of the $40^{\text {th }}$ interscience conference on Antimicrobial Agents and chemotherapy: Toronto Ontario, Canada. September 17-20, 2000. Abstract 1906.

2. Miller R HIV associated respiratory diseases. Lancet 1996; 348:307-312.

3. Price RW. Neurological complications of HIV infection. Lancet 1996; 348:445-452.

4. Tschachler E., Bergstresser PR., Stingi G. HIV related skin diseases. Lancet 1996; 348: 659-663.

5. Fauci A.S. Lave H.C. Human immunodeficiency virus (HIV) disease. AIDS related diseases in Fauci A.S. Braunwald E. Isselbacker K.J. et al (eds) Harrison principles of internal medicine $14^{\text {th }}$ edition McGraw Hill Co. 1998: 1835-1837

6. Ayoola E.A. Infections disease in Africa. Infection 1987; 273(15):153-

7. William M.J. Hepatitis B virus infection. N.Engl. J. Med. 1997; 337:1733-1743

8. Dienstrug J.L.V. Isselbacher K.J. Acute viral hepatitis in: Fauci A.S. Bramwald 
E. Issebacher K.J. (eds). Harrision's principles of internal medicine $14^{\text {th }}$ ed. McGraw Hill co.1998:1677-1692.

9. Bertian Auvret, Ron Ballard, Catherine Campbell et al.High prevalence of HIV infection among younth in a South Africa mining Town is associated with HSV-2 seropositivity and sexual behaviour. AIDS 2001;15:1-2

10. Summary finding from the 2005 HIV/Syphilis sentinel sero-prevalence survey in Nigeria information for policy makers: 1-4.

11. Schulz T.R. Boshof .C. Ho. Weiss R.A. HIV infection and Neoplasia Lancet. 1996; 348:587-91.

12. Edwards CRW Boucher. J.A.D. Haslett C. Chilvers E. (eds) principle and practice of medicine. Chuchill Livingstone. 1995.85-94.

13. Oyejide CO. Health research methods for developing countries scientists .ISBN 9h8-195.0442:1998:59-63.

14. Ahmed S.D. Cuevas .L.E. Brabin R.J. Kazembe .P. et al. Seroprevalence of hepatitis B. and C. and HIV in Malawian pregnant women .J. Infect. 1998; 37 (3): 248-257.

15. Altra M.A. prevalence of hepatitis B in Egypt and Africa. Antivither. 1998; 3(3): 1-9.
16. Sirisena .N.D. Njoku M.O. Idoko, J.A.Hepatitis B. surface antigen in $\mathrm{p}$ a t i e n t s w it h h u m a n immunodeficiency virus 1(HIV-1) infection in Jos, Nigeria. Nigerian medical practitioner 2002; 41:18-20.

17. Sirisena. N.D. the prevalence and clinical pattern of human immunodeficiency virus 1(HIV1) infection in Nigerian prisoners. Nigerian medical practitioner 2002; 41:8-14

18. Agbaji OO. The prevalence of hepatitis $B$ and $C$ virus infections in Hemodialysis patients in Jos Un i v e r s it y $\mathrm{Te}$ a $\mathrm{c}$ h i n g Hospital(JUTH), Jos . FMCP Dissertation. 2000.

19. Maynard J.E. control of hepatitis a n d liver disease. Zuckerman A.J. (ed). New York. Alan R. Liss, 1988; 167-168.

20. Halim N.K.D. Ajayi O.I; Risk factors and seroprevalence of hepatitis C Antibody in blood donors in Nigeria. East Africal medical Journal. 2000; 77(8):410- 412.

21. Jimeuez-fabregaX. CarballoAlmeida A. Batalla-marlinnez C. et al. The prevalence of hepatitis. B and $\mathrm{C}$ co-infection and HIV intravenous drug users AtenPrimera. 1999; 24(6): 368-371 